\title{
Clinical Outcomes of Endoscopic Resection for Low-Grade Dysplasia and High-Grade Dysplasia on Gastric Pretreatment Biopsy: Korea ESD Study Group
}

\author{
Jung Won Jeon ${ }^{1}$, Soo Jin Kim², Jae Young Jang ${ }^{3}$, Sun-Moon Kim4, Chul-Hyun Lim ${ }^{5}$, Jae Myung Park ${ }^{5}$, \\ Su Jin Hong ${ }^{6}$, Chan Gyoo Kim ${ }^{7}$, Seong Woo Jeon ${ }^{8}$, Si Hyung Lee ${ }^{9}$, Jae Kyu Sung ${ }^{10}$, and Gwang Ho Baik ${ }^{11,12}$ \\ 1Department of Internal Medicine, Kyung Hee University Hospital at Gangdong, Kyung Hee University College of Medicine, \\ ${ }^{2}$ Institute of Medical Science, Kyung Hee University Hospital at Gangdong, ${ }^{3}$ Department of Internal Medicine, Kyung Hee University \\ College of Medicine, Seoul, ${ }^{4}$ Department of Internal Medicine, Konyang University College of Medicine, Daejeon, ${ }^{5}$ Department of \\ Internal Medicine, College of Medicine, The Catholic University of Korea, Seoul, ${ }^{6}$ Department of Internal Medicine, Soonchunhyang \\ University College of Medicine, Bucheon, ${ }^{7}$ Center for Gastric Cancer, Research Institute and Hospital, National Cancer Center, \\ Goyang, ${ }^{8}$ Department of Internal Medicine, School of Medicine, Kyungpook National University, ${ }^{9}$ Department of Internal Medicine, \\ Yeungnam University College of Medicine, Daegu, ${ }^{10}$ Department of Internal Medicine, Chungnam National University School of \\ Medicine, Daejeon, ${ }^{11}$ Department of Internal Medicine, Hallym University College of Medicine, Chuncheon Sacred Heart Hospital, and \\ ${ }^{12}$ Institute for Liver and Digestive Diseases, Hallym University College of Medicine, Chuncheon, Korea
}

\section{Article Info}

Received July 30, 2019

Revised March 26, 2020

Accepted April 11, 2020

Published online August 10, 2020

Corresponding Author

Gwang Ho Baik

ORCID https://orcid.org/0000-0003-1419-7484

E-mail baikgh@hallym.or.kr
Background/Aims: Some cases of gastric low-grade dysplasia (LGD) and high-grade dysplasia (HGD) on forceps biopsy (FB) are diagnosed as gastric cancer (GC) after endoscopic resection (ER). This study aims to evaluate the clinical outcomes of ER for gastric LGD and HGD on pretreatment FB and to identify the factors that predict pathologic upstaging to GC.

Methods: Patients who underwent ER for LGD and HGD on pretreatment FB from March 2005 to February 2018 in 14 hospitals in South Korea were enrolled, and the patients' medical records were reviewed retrospectively.

Results: This study included 2,150 cases of LGD and 1,534 cases of HGD diagnosed by pretreatment FB. In total, 589 of 2,150 LGDs (27.4\%) were diagnosed as GC after ER. Helicobacter pylori infection, smoking history, tumor location in the lower third of the stomach, tumor size $>10$ $\mathrm{mm}$, depressed lesion, and ulceration significantly predicted GC. A total of 1,115 out of 1,534 HGDs (72.7\%) were diagnosed with GC after ER. Previous history of GC, H. pylori infection, smoking history, tumor location in the lower third of the stomach, tumor size $>10 \mathrm{~mm}$, depressed lesion, and ulceration were significantly associated with GC. As the number of risk factors predicting $G C$ increased in both LGD and HGD on pretreatment $F B$, the rate of upstaging to $G C$ after ER increased.

Conclusions: A substantial proportion of LGDs and HGDs on pretreatment FB were diagnosed as GC after ER. Accurate ER procedures such as endoscopic submucosal dissection should be recommended in cases of LGD and HGD with factors predicting pathologic upstaging to GC. (Gut Liver 2021;15:225-231)

Key Words: Low-grade dysplasia; High-grade dysplasia; Endoscopic resection; Gastric cancer; Risk factors

\section{INTRODUCTION}

In the latest published global cancer statistics, gastric cancer (GC) is ranked as the third most common cause for cancer-related mortality worldwide. ${ }^{1}$ Early detection and proper management of GC and precancerous lesions are crucial to improving GC-related mortality. Gastric dysplasia is regarded as a precancerous lesion. ${ }^{2}$ The risk of carcinoma generally increases with the histological grade of the dysplasia (low to high grade). According to the revised 
Vienna classification, ${ }^{3}$ gastric low-grade dysplasia (LGD) is classified as category 3 , and endoscopic resection (ER) or regular follow-up examination is recommended. Category 4 is defined as noninvasive high-grade neoplasia. Category 4 is further divided into category 4.1 defined as high-grade dysplasia (HGD); 4.2, noninvasive carcinoma (carcinoma in situ); 4.3, suspicion of invasive carcinoma based on the degree of structural or cytological atypia of the neoplastic glands; and 4.4, intramucosal carcinoma. It is strongly recommended that gastric HGD, which is highly predictive of carcinoma be treated with ER or surgical resection.

Endoscopic examination is useful in detecting gastric neoplasia in its early stage, and pathological examination of endoscopic forceps biopsy (FB) is the gold standard for an accurate diagnosis. However, cases in which the initial pathological diagnosis on pretreatment FB is corrected after ER are frequently found, due to the difficulty of making solid diagnosis based on small biopsy specimens. ${ }^{4}$ For this reason, GC may be underdiagnosed as LGD or HGD on pretreatment FB. Previous studies have reported that $12.1 \%$ to $63 \%$ of LGD lesions are upgraded to HGD or GC after ER ${ }^{5-15}$ In addition, gastric HGD has been shown to be GC in about $27 \%$ to $80 \%$ of cases after ER. ${ }^{16-18}$

In this study, we aimed to evaluate the clinical outcomes of ER for gastric LGDs and HGDs on pretreatment FB, using the multicenter large-scale endoscopic submucosal dissection (ESD) registry database. In addition, we investigated the factors predicting the pathologic upstage to GC.

\section{MATERIALS AND METHODS}

\section{Study population}

We identified and reviewed cases which were treated with ER for LGD and HGD on pretreatment FB and involved in the Korean ESD registry database- an online registry created in 2015. It is a project which collects ESD or endoscopic mucosal resection (EMR) data from multiple centers, each representing its district in South Korea, and is under the control of Korean Society of Gastrointestinal Endoscopy. This registry contains clinical information, endoscopic findings, pathologic results, therapeutic outcomes, and follow-up data related to ER for gastric neoplasms.

This study included patients who had undergone ER for gastric LGD and HGD on pretreatment FB at 14 university hospitals in South Korea from March 2005 to February 2018. Medical records of patients involved in this study were retrospectively reviewed. Patients' data include age, sex, family history of GC, previous history of GC, the presence of hypertension or diabetes mellitus, aspirin use, smoking history, and Helicobacter pylori infection.

\section{Endoscopic and pathologic evaluation}

Endoscopic reports of all enrolled lesions were reviewed to determine the features of lesions. The Japanese classification of GC was used to describe the location of lesions. ${ }^{19}$ The Paris classification was used to define the gross types of superficial lesions, which were divided into elevated, flat, or depressed..$^{20}$ Ulcers which were defined as breaks in the mucosal surface $>5 \mathrm{~mm}$ in size with depth to the submucosa, were also evaluated.

Pathologic reports of the resected tissues, which were reported by experienced pathologists in hospitals involved in this study, were reviewed. All of the lesions were classified as gastrointestinal epithelial neoplasia following the Vienna classification. ${ }^{2}$

\section{Statistical analysis}

Univariate analysis with the chi-square test or Fisher exact test for categorical variables and the Student t-test for continuous variables were performed. Multivariate analysis with a multiple logistic regression model was performed

Table 1. Baseline Characteristics

\begin{tabular}{lcc}
\multicolumn{1}{c}{ Characteristics } & $\begin{array}{c}\text { LGD on } \\
\text { pretreatment FB } \\
(\mathrm{n}=2,150)\end{array}$ & $\begin{array}{c}\text { HGD on } \\
\text { pretreatment FB } \\
(\mathrm{n}=1,534)\end{array}$ \\
\hline Age, yr & $63.89 \pm 9.36$ & $65.24 \pm 9.17$ \\
Male sex & $1,493(69.4)$ & $1,113(72.6)$ \\
Family history of GC & $73(3.4)$ & $60(3.9)$ \\
Previous history of GC & $43(2.0)$ & $70(4.6)$ \\
Helicobacter pylori infection & $570(26.5)$ & $371(24.2)$ \\
Smoking history & $379(17.6)$ & $527(34.4)$ \\
Hypertension & $748(34.8)$ & $579(37.7)$ \\
Diabetes mellitus & $338(15.7)$ & $270(17.6)$ \\
Aspirin use & $232(10.8)$ & $180(11.7)$ \\
Tumor location & & \\
Lower third of stomach & $1,217(56.6)$ & $952(62.1)$ \\
Middle third of stomach & $789(36.7)$ & $461(30.0)$ \\
Upper third of stomach & $144(6.7)$ & $121(7.9)$ \\
Tumor size, mm & $13.94 \pm 11.28$ & $15.89 \pm 11.92$ \\
Gross type & & \\
Elevated & $1,249(58.1)$ & $851(55.5)$ \\
Flat & $692(32.2)$ & $322(21.0)$ \\
Depressed & $209(9.7)$ & $361(23.5)$ \\
Ulcer & $91(4.2)$ & $220(14.3)$ \\
Pathologic concordance & $1,038(48.3)$ & $328(21.4)$ \\
Pathologic downstage & $131(6.1)$ & $91(5.9)$ \\
Pathologic upstage to GC & $589(27.4)$ & $1,115(72.7)$ \\
Endoscopic resection method & & \\
EMR & $996(46.3)$ & $405(26.4)$ \\
ESD & $1,154(53.7)$ & $1,129(73.6)$ \\
En bloc resection & $2,085(97.1)$ & $1,486(96.9)$ \\
Complete resection & $2,092(97.4)$ & $1,494(97.4)$ \\
\hline Dar & & \\
\hline & &
\end{tabular}

Data are presented as mean \pm SD or number $(\%)$.

LGD, low-grade dysplasia; HGD, high-grade dysplasia; FB, forceps biopsy; GC, gastric cancer; EMR, endoscopic mucosal resection; ESD, endoscopic submucosal dissection. 
to identify risk factors for GC. The $\mathrm{p}<0.05$ was considered statistically significant. Statistical calculations were performed with SPSS version 21.0 for Windows (IBM Corp., Armonk, NY, USA).

\section{Ethics statement}

This study was conducted according to the principles expressed in the Declaration of Helsinki, and approved by the Institutional Review Board of Chuncheon Sacred Heart Hospital (IRB number: 2016-87).

\section{RESULTS}

From March 2005 to February 2018, 2,277 LGD lesions and 1,620 HGD lesions, which were managed by ER, were enrolled in the Korean ESD registry database. Among these, 127 LGD lesions and 86 HGD lesions were excluded because they were incomplete to be used as valid data. Ultimately, the data from 2,150 LGD lesions and 1,534 HGD lesions were analyzed.

Table 1 shows the clinical and endoscopic characteristics of LGD and HGD on pretreatment FB. The patients' mean age was $63.89 \pm 9.36$ years in LGD group and 65.24 \pm 9.17 years in HGD group. There were 1,493 (69.4\%) males in the LGD group and 1,113 (72.6\%) in HGD group. Fortythree cases $(2.0 \%)$ in the LGD group and 70 cases (4.6\%) in HGD group had a previous history of GC. We identified incidence rate of $H$. pylori infection as $26.5 \%(570 / 2,150)$ in the LGD group and $24.2 \%(371 / 1,354)$ in the HGD group. The 379 cases (17.6\%) in the LGD group and 527 cases (34.4\%) in the HGD group had past or current smoking history. Most lesions were elevated in gross type (58.1\% in the LGD group and $55.5 \%$ in the HGD group) and located in the lower third of stomach $56.6 \%$ in the LGD group and $62.1 \%$ in the HGD group). Ninety-one cases (4.2\%) in the LGD group and 220 cases (14.3\%) in the HGD group showed ulceration. The 1,154 cases (53.7\%) in the LGD group and 1,129 cases (73.6\%) in the HGD group were managed by ESD. The en bloc resection rate was $97.1 \%$ in the LGD group and $96.9 \%$ in the HGD group. The complete resection rate was $97.4 \%$ in both groups.

Pathologic concordance rate was $48.3 \%(1,038 / 2,150)$ in the LGD group and $21.4 \%(328 / 1,534)$ in the HGD group. The 589 of 2,150 cases (27.4\%) in the LGD group and 1,115 of 1,534 cases $(72.7 \%)$ in the HGD group showed pathologic upstage to GC after ER.

Table 2 shows the factors for upgrade diagnosis to GC of LGD in univariate and multivariate analyses. Multivariate analysis revealed that $H$. pylori infection (absence of $H$. pylori infection compared with $H$. pylori infection; odds ratio [OR], 0.686; 95\% confidence interval [CI], 0.498 to $0.945 ; \mathrm{p}=0.021$ ), smoking history ( $\mathrm{OR}, 4.928 ; 95 \% \mathrm{CI}$, 3.290 to $7.383 ; \mathrm{p}<0.001$ ), tumor location in the lower third of the stomach (middle third compared with lower third: OR, $0.654 ; 95 \% \mathrm{CI}, 0.487$ to $0.878 ; \mathrm{p}=0.005$ ), tumor size of $>10 \mathrm{~mm}$ (OR, 3.467; 95\% CI, 2.571 to 4.675 ; $\mathrm{p}<0.001$ ), depressed lesion (OR, 3.270; 95\% CI, 2.067 to 5.171;

Table 2. Risk Factors for Upgrading of LGD to GC in Univariate and Multivariate Analysis

\begin{tabular}{|c|c|c|c|c|c|c|}
\hline \multirow{2}{*}{ Factor } & \multicolumn{3}{|c|}{ Univariate analysis } & \multicolumn{3}{|c|}{ Multivariate analysis } \\
\hline & $\mathrm{OR}$ & $95 \% \mathrm{Cl}$ & $\mathrm{p}$-value & $\mathrm{OR}$ & $95 \% \mathrm{Cl}$ & p-value \\
\hline Age $>65 y r$ & 1.234 & $1.012-1.504$ & 0.038 & 1.102 & $0.827-1.469$ & 0.508 \\
\hline Male sex & 1.150 & $0.924-1.430$ & 0.034 & 0.808 & $0.590-1.107$ & 0.185 \\
\hline Family history of GC & 0.926 & $0.451-1.904$ & 0.835 & & & \\
\hline Absence of previous history of GC & 2.421 & $1.211-4.839$ & 0.012 & 0.838 & $0.340-2.066$ & 0.701 \\
\hline Absence of Helicobacter pylori infection & 0.755 & $0.579-0.984$ & 0.038 & 0.686 & $0.498-0.945$ & 0.021 \\
\hline Smoking history & 5.374 & $4.149-6.960$ & $<0.001$ & 4.928 & $3.290-7.383$ & $<0.001$ \\
\hline Hypertension & 1.130 & $0.919-1.389$ & 0.247 & 1.264 & $0.935-1.709$ & 0.128 \\
\hline Diabetes mellitus & 1.113 & $0.846-1.163$ & 0.445 & 0.738 & $0.497-1.096$ & 0.132 \\
\hline Aspirin use & 0.815 & $0.590-1.127$ & 0.217 & 0.949 & $0.601-1.498$ & 0.821 \\
\hline \multicolumn{7}{|l|}{ Tumor location } \\
\hline Lower third of stomach (reference) & 1.000 & & & 1.000 & & \\
\hline Middle third of stomach & 0.785 & $0.635-0.971$ & 0.025 & 0.654 & $0.487-0.878$ & 0.005 \\
\hline Upper third of stomach & 0.963 & $0.651-1.426$ & 0.851 & 1.161 & $0.636-2.121$ & 0.626 \\
\hline Tumor size $>10 \mathrm{~mm}$ & 2.730 & $2.200-3.389$ & $<0.001$ & 3.467 & $2.571-4.675$ & $<0.001$ \\
\hline \multicolumn{7}{|l|}{ Gross type } \\
\hline Elevated (reference) & 1.000 & & & 1.000 & & \\
\hline Flat & 0.468 & $0.368-0.596$ & $<0.001$ & 0.790 & $0.577-1.084$ & 0.144 \\
\hline Depressed & 2.320 & $1.661-3.239$ & $<0.001$ & 3.270 & $2.067-5.171$ & $<0.001$ \\
\hline Absence of ulcer & 0.226 & $0.176-0.291$ & $<0.001$ & 0.203 & $0.147-0.282$ & $<0.001$ \\
\hline
\end{tabular}

LGD, low-grade dysplasia; GC, gastric cancer; OR, odds ratio; $\mathrm{Cl}$, confidence interval. 
$\mathrm{p}<0.001$ ), and ulceration (absence of ulceration compared with ulceration: OR, 0.203 ; $95 \% \mathrm{CI}, 0.147$ to 0.282 ; $\mathrm{p}<0.001$ ) were significant predictive factors of the upstage diagnosis to GC of LGD. Table 3 and Fig. 1 show the effect of the presence of 0-6 risk factors on upstage diagnosis to GC of LGD. An increase in the number of risk factors was significantly associated with an increasing rate of upstage diagnosis to GC of LGD.

Table 4 shows the factors for upgrade diagnosis to GC of HGD in univariate and multivariate analyses. Multivariate analysis revealed that previous history for GC (absence of previous history for GC compared with previous history of GC: OR, $0.459 ; 95 \% \mathrm{CI}, 0.257$ to $0.819 ; \mathrm{p}=0.008), H$.

Table 3. The Effect of the Presence of 0-6 Risk Factors upon Upstaging of $L G D$ to $G C$

\begin{tabular}{cccc}
\hline $\begin{array}{c}\text { No. of } \\
\text { risk factors }\end{array}$ & $\begin{array}{c}\text { LGD or } \\
\text { downgrade } \\
\text { diagnostic group } \\
(\mathrm{n}=1,169)\end{array}$ & $\begin{array}{c}\text { GC group } \\
\text { (n=589) }\end{array}$ & $\begin{array}{c}\text { Total } \\
\text { (n=1,778) }\end{array}$ \\
\hline 0 & 3 & 0 & 3 \\
1 & 65 & 1 & 66 \\
2 & 394 & 39 & 433 \\
3 & 462 & 162 & 624 \\
4 & 222 & 234 & 456 \\
5 & 23 & 134 & 157 \\
6 & 0 & 19 & 19 \\
\hline
\end{tabular}

Date are presented as number. Risk factors: Helicobacter pylori infection, smoking history, tumor location in the middle third of the stomach, tumor lesion size $>10 \mathrm{~mm}$, depressed lesion, and ulcer. LGD, low-grade dysplasia; GC, gastric cancer. pylori infection (absence of $H$. pylori infection compared with $H$. pylori infection: OR, 0.585 ; $95 \% \mathrm{CI}, 0.430$ to 0.795 ; $\mathrm{p}=0.001)$, smoking history (OR, 2.527; $95 \% \mathrm{CI}, 1.790$ to 3.567; $\mathrm{p}<0.001$ ), tumor location in lower third of the stomach (upper third compared with lower third: OR, 0.544; 95\% CI, 0.332 to $0.890 ; \mathrm{p}=0.015$ ), tumor size of $>10 \mathrm{~mm}$ (OR, 1.934; 95\% CI, 1.438 to 2.600; $\mathrm{p}<0.001$ ), depressed lesion (OR, 2.551; 95\% CI, 1.731 to 3.758; $\mathrm{p}<0.001$ ), and ul-

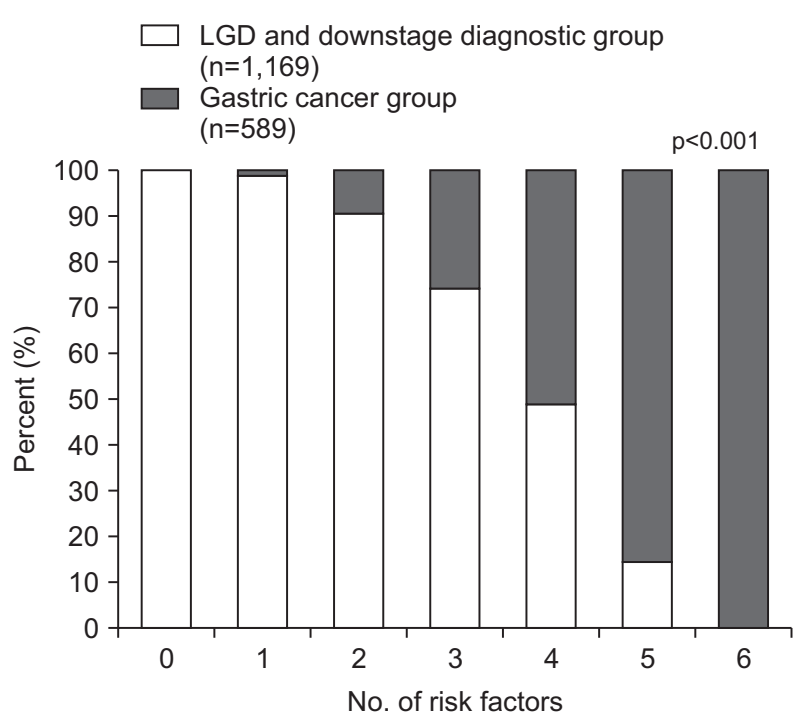

Fig. 1. The effect of the presence of $0-6$ risk factors upon upstaging of a low-grade dysplasia (LGD) diagnosis to gastric cancer. Risk factors: Helicobacter pylori infection, smoking history, tumor location in the lower third of the stomach, tumor lesion size $>10 \mathrm{~mm}$, depressed lesion, and ulcer.

Table 4. Factors Associated with Upstaging of HGD to GC in Univariate and Multivariate Analysis

\begin{tabular}{|c|c|c|c|c|c|c|}
\hline \multirow{2}{*}{ Factor } & \multicolumn{3}{|c|}{ Univariate analysis } & \multicolumn{3}{|c|}{ Multivariate analysis } \\
\hline & OR & $95 \% \mathrm{Cl}$ & $\mathrm{p}$-value & OR & $95 \% \mathrm{Cl}$ & $\mathrm{p}$-value \\
\hline Age $>65 y r$ & 0.868 & $0.691-1.090$ & 0.223 & 0.789 & $0.600-1.039$ & 0.092 \\
\hline Male sex & 1.307 & $1.020-1.675$ & 0.034 & 0.862 & $0.625-1.189$ & 0.366 \\
\hline Family history of GC & 0.714 & $0.365-1.398$ & 0.326 & & & \\
\hline Absence of previous history of GC & 0.622 & $0.378-1.023$ & 0.061 & 0.459 & $0.257-0.819$ & 0.008 \\
\hline Absence of Helicobacter pylori infection & 0.655 & $0.197-0.863$ & 0.003 & 0.585 & $0.430-0.795$ & 0.001 \\
\hline Smoking history & 2.399 & $1.844-3.121$ & $<0.001$ & 2.527 & $1.790-3.567$ & $<0.001$ \\
\hline Hypertension & 1.016 & $0.806-1.281$ & 0.892 & & & \\
\hline Diabetes mellitus & 1.115 & $0.826-1.506$ & 0.475 & & & \\
\hline Aspirin use & 0.944 & $0.668-1.334$ & 0.744 & & & \\
\hline \multicolumn{7}{|l|}{ Tumor location } \\
\hline Lower third of stomach (reference) & 1.000 & & & 1.000 & & \\
\hline Middle third of stomach & 0.846 & $0.660-1.084$ & 0.185 & 0.849 & $0.628-1.147$ & 0.285 \\
\hline Upper third of stomach & 0.702 & $0.468-1.053$ & 0.087 & 0.544 & $0.332-0.890$ & 0.015 \\
\hline Tumor size $>10 \mathrm{~mm}$ & 1.293 & $1.015-1.649$ & 0.038 & 1.934 & $1.438-2.600$ & $<0.001$ \\
\hline \multicolumn{7}{|l|}{ Gross type } \\
\hline Elevated (reference) & 1.000 & & & 1.000 & & \\
\hline Flat & 0.874 & $0.661-1.156$ & 0.346 & 1.048 & $0.752-1.461$ & 0.780 \\
\hline Depressed & 2.430 & $1.747-3.381$ & $<0.001$ & 2.551 & $1.731-3.758$ & $<0.001$ \\
\hline Absence of ulcer & 0.627 & $0.484-0.812$ & $<0.001$ & 0.537 & $0.388-0.743$ & $<0.001$ \\
\hline
\end{tabular}

$\mathrm{HGD}$, high-grade dysplasia; $\mathrm{GC}$, gastric cancer; $\mathrm{OR}$, odds ratio; $\mathrm{Cl}$, confidence interval. 
Table 5. The Effect of the Presence of 0-7 Risk Factors upon Upstaging of HGD to GC

\begin{tabular}{cccc}
\hline $\begin{array}{c}\text { No. of risk } \\
\text { factors }\end{array}$ & $\begin{array}{c}\text { HGD or } \\
\text { downgrade } \\
\text { diagnostic group } \\
(\mathrm{n}=419)\end{array}$ & $\begin{array}{c}\text { GC group } \\
(\mathrm{n}=1,115)\end{array}$ & $\begin{array}{c}\text { Total } \\
(\mathrm{n}=1,534)\end{array}$ \\
\hline 0 & 1 & 0 & 1 \\
1 & 9 & 3 & 12 \\
2 & 54 & 63 & 117 \\
3 & 175 & 266 & 441 \\
4 & 120 & 445 & 565 \\
5 & 51 & 246 & 297 \\
6 & 9 & 84 & 93 \\
7 & 0 & 8 & 8 \\
\hline
\end{tabular}

Date are presented as number. Risk factors: previous history of GC, Helicobacter pylori infection, smoking history, tumor location in the upper third of the stomach, tumor lesion size $>10 \mathrm{~mm}$, depressed lesion, and ulcer.

HGD, high-grade dysplasia; GC, gastric cancer.

ceration (absence of ulceration compared with ulceration: OR, $0.537 ; 95 \%$ CI, 0.388 to 0.743 ; $\mathrm{p}<0.001$ ) were significant predictive factors for upstage diagnosis to GC of HGD. Table 5 and Fig. 2 show the effect of the presence of $0-7$ risk factors on upstage diagnosis to GC of HGD. An increase in the number of risk factors was significantly associated with an increasing rate of upstage diagnosis to GC of HGD.

\section{DISCUSSION}

The specimens obtained by endoscopic FB-an essential diagnostic tool for gastric superficial neoplasms, may not be representative of the entire lesion. ${ }^{5}$ The possible reasons for this discrepancy may be as follows: ${ }^{17}$ (1) FB samples are small in size, and therefore do not represent the entire lesion; (2) cancer is sometimes hidden in other parts of the lesion; or (3) the atypia of adenoma and adenocarcinoma is too subtle to detect in a small biopsy specimen.

In previous studies, it has been reported that $12.1 \%$ to $63 \%$ of LGD lesions are upgraded to HGD or GC after ER. ${ }^{5-15}$ In 2,150 LGD cases of our study, 981 cases (45.6\%) showed diagnostic upgrade to HGD or GC after ER, which is similar to the results of previous studies. Diagnostic upgrades to HGD and GC were 392 cases (18.2\%) and 589 cases (27.4\%), respectively. Likewise, in the case of HGD, previous studies showed that gastric HGD on pretreatment FB was diagnosed as GC in about $27.6 \%$ to $80 \%$ of cases after ER. ${ }^{16-18}$ Diagnostic upgrade to GC was found in $72.7 \%$ of cases in our study, which is similar to the results of previous studies.

Previous studies reported that several endoscopic findings were associated with the risk of GC. ${ }^{17,21}$ We investigat-
HGD and downstage diagnostic group $(n=419)$

$\square$ Gastric cancer group $(n=1,115)$

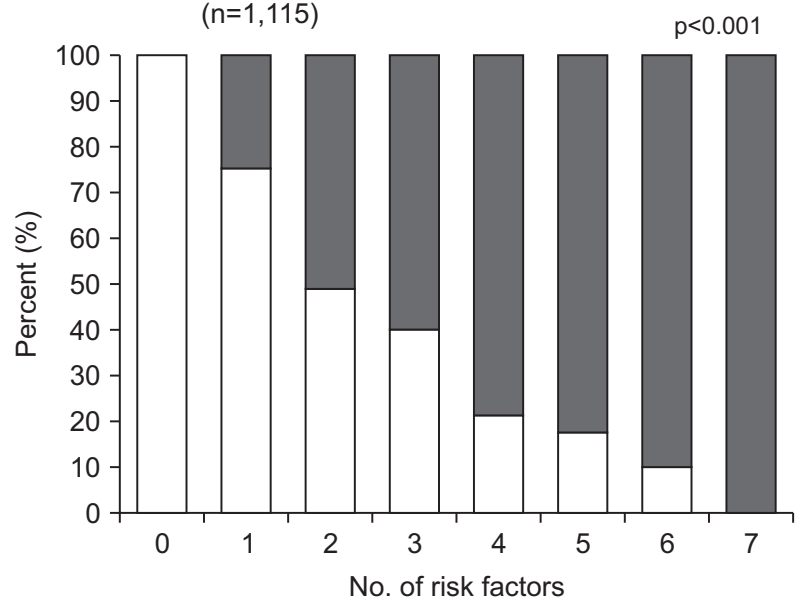

Fig. 2. The effect of the presence of 0-7 risk factors upstaging of a high-grade dysplasia (HGD) diagnosis to gastric cancer (GC). Risk factors: previous history of GC, Helicobacter pylori infection, smoking history, tumor location in the lower third of the stomach, tumor lesion size $>10 \mathrm{~mm}$, depressed lesion, and ulcer.

ed the risk factors for GC in LGD and HGD, respectively. In addition, we investigated endoscopic findings as well as clinical characteristics when investigating risk factors for GC. In the case of LGD, previous articles examined factors that could predict HGD as well as GC..$^{5,8,12,13}$ Although the modified Vienna classification of epithelial neoplasia is used widely, ${ }^{3}$ differences exist between the Asian and Western pathologists regarding the histological criteria in grading dysplasia. ${ }^{22}$ Moreover, Western gastroenterologists are more interested in GC than HGD or LGD. For these reasons, this study only focused on risk factors for GC.

Kang et al. ${ }^{13}$ reported that the rate of diagnostic upstage to HGD or GC of LGD increased as the number of risk factors increased. In the present study, we evaluated the association between the total number of risk factors and the incidence of diagnostic upstage to GC in LGD and HGD respectively. As the number of risk factors increased in both LGD and HGD, the rate of upstage diagnosis to GC also increased.

Our study showed that the location in lower third of LGD and HGD, compared with the location in middle third of LGD and upper third of HGD respectively, was associated with histologic upgrade to GC. We assume that investigators could miss LGD or HGD in blind spots of middle or upper third of stomach before the lesions change to cancer and observed clearly.

When we conducted the study, we used the ESD registry database-an online registry created in 2015. ESD registry involved more than 9,000 cases. Of them, we identified 
and reviewed cases which were treated with ER for LGD and HGD on pretreatment FB. We knew that the percent of $H$. pylori infection was low after analysis of data. We assume this is the limitation of retrospective study. Although The percent of $H$. pylori infection was low and the bias was thought to be involved, we think the analysis which conducted with data showing bias would be make sense to assure the risk of $H$. pylori for GC.

In recent years, ESD has become an accepted curative treatment modality for the treatment of early GC. ${ }^{23}$ Therefore, it is important to completely remove the lesions identified to be LGD or HGD on pretreatment FB, if risk factors predicting upstage diagnosis to GC are identified. Compared to EMR, ESD can effectively remove LGD or HGD lesions without marginal involvement of the lesion. ESD increases the en bloc resection rate for lesions $>10$ mm, compared with EMR. ${ }^{5}$ The Korean National Health Insurance System provides insurance for ESD of LGD and HGD lesions $>15 \mathrm{~mm}$. In South Korea, until recently, LGD or HGD lesions between $10 \mathrm{~mm}$ and $15 \mathrm{~mm}$ were treated with modified EMR involving EMR after precutting or EMR using scope detaching cap in the tip instead of conventional EMR. According to our study, the insurance criteria for the size of the lesion in South Korea may need to be changed. In addition to the size of the lesion, other risk factors involving $H$. pylori infection, smoking history, tumor location, depressed lesion and the presence of ulcer were significantly associated with the upgrade diagnosis to GC. Therefore, it is necessary to expand the coverage of medical insurance for ESD in the cases of LGD and HGD involving risk factors predicting the upgrade diagnosis to GC in South Korea.

Fourteen hospitals in South Korea participated in this study. The researchers participated in this study of each hospital shared respective definitions for the data that they wanted to collect. They checked the low data of the patients enrolled in their hospitals and filled up the missing data as much as possible. Despite these efforts, this study has limitations. This is retrospective study, so for example the percentage of $H$. pylori infection in individuals involved in this study is low. We could not guarantee that all pathologists of 14 hospitals involved in this study show concordance of pathologic diagnosis. Although the definitions for clinical data have been shared in advance, not all data may be consistent in terms of definitions. The gross size and type for the lesions confirmed by the endoscopic photographs may vary depending on the examiner. ESD registry did not involve the factor for the color change of the lesion, such as reddish color change of mucosa. The presence of an ulcer can also vary from person to person. However, despite these limitations, this study is a multi- center study conducted by the most influential institute in the field of gastrointestinal endoscopy in South Korea. In addition, this study involves many subjects.

In conclusion, a substantial proportion of LGD and HGD on pretreatment FB were diagnosed as GC after ER. As the number of risk factors predicting GC increased in both LGD and HGD on pretreatment FB, the rate of upstage diagnosis to GC after ER also increased. Therefore, accurate ER such as ESD should be recommended in cases of LGD and HGD with factors predicting pathologic upstage to GC.

\section{CONFLICTS OF INTEREST}

No potential conflict of interest relevant to this article was reported.

\section{AUTHOR CONTRIBUTIONS}

Study concept and design: J.W.J., G.H.B. Statistical analysis: S.J.K. Study supervision: S.J.H. Data acquisition: J.Y.J., S.M.K., C.H.L., J.M.P., C.G.K., S.W.J., S.H.L., J.K.S. Writing - original draft: J.W.J. Writing - review and editing: G.H.B., J.W.J., S.J.K. Approval of final manuscript: all authors.

\section{ORCID}

Jung Won Jeon Soo Jin Kim Jae Young Jang Sun-Moon Kim Chul-Hyun Lim Jae Myung Park Su Jin Hong Chan Gyoo Kim Seong Woo Jeon Si Hyung Lee Jae Kyu Sung Gwang Ho Baik
https://orcid.org/0000-0003-3087-086X https://orcid.org/0000-0002-1676-8060 https://orcid.org/0000-0002-7930-1468 https://orcid.org/0000-0002-0436-3381 https://orcid.org/0000-0002-8347-8979 https://orcid.org/0000-0002-1534-7467 https://orcid.org/0000-0003-2012-0360 https://orcid.org/0000-0002-1651-2559 https://orcid.org/0000-0002-9539-9389 https://orcid.org/0000-0001-7221-7506 https://orcid.org/0000-0002-9068-624X https://orcid.org/0000-0003-1419-7484

\section{REFERENCES}

1. Bray F, Ferlay J, Soerjomataram I, Siegel RL, Torre LA, Jemal A. Global cancer statistics 2018: GLOBOCAN estimates of incidence and mortality worldwide for 36 cancers in 185 countries. CA Cancer J Clin 2018;68:394-424.

2. Dixon MF. Gastrointestinal epithelial neoplasia: Vienna re- 
visited. Gut 2002;51:130-131.

3. Stolte M. The new Vienna classification of epithelial neoplasia of the gastrointestinal tract: advantages and disadvantages. Virchows Arch 2003;442:99-106.

4. Nishida T, Tsutsui S, Kato M, et al. Treatment strategy for gastric non-invasive intraepithelial neoplasia diagnosed by endoscopic biopsy. World J Gastrointest Pathophysiol 2011;2:93-99.

5. Choi CW, Kim HW, Shin DH, et al. The risk factors for discrepancy after endoscopic submucosal dissection of gastric category 3 lesion (low grade dysplasia). Dig Dis Sci 2014;59:421-427.

6. Choi CW, Kang DH, Kim HW, Park SB, Kim S, Cho M. Endoscopic submucosal dissection as a treatment for gastric adenomatous polyps: predictive factors for early gastric cancer. Scand J Gastroenterol 2012;47:1218-1225.

7. Zhu LY, Dai J, Zhao YJ, Xue HB, Ge ZZ, Li XB. Endoscopic resection for gastric epithelial neoplasia: how to solve pathological discrepancy and achieve curative resection? J Dig Dis 2013;14:231-237.

8. Lim H, Jung HY, Park YS, et al. Discrepancy between endoscopic forceps biopsy and endoscopic resection in gastric epithelial neoplasia. Surg Endosc 2014;28:1256-1262.

9. Hwang JW, Bae YS, Kang MS, et al. Predicting pre- and postresectional histologic discrepancies in gastric low-grade dysplasia: a comparison of white-light and magnifying endoscopy. J Gastroenterol Hepatol 2016;31:394-402.

10. Lee JH, Min YW, Lee JH, et al. Diagnostic group classifications of gastric neoplasms by endoscopic resection criteria before and after treatment: real-world experience. Surg Endosc 2016;30:3987-3993.

11. Takao M, Kakushima N, Takizawa K, et al. Discrepancies in histologic diagnoses of early gastric cancer between biopsy and endoscopic mucosal resection specimens. Gastric Cancer 2012;15:91-96.

12. Cho SJ, Choi IJ, Kim CG, et al. Risk of high-grade dysplasia or carcinoma in gastric biopsy-proven low-grade dysplasia: an analysis using the Vienna classification. Endoscopy 2011;43:465-471.
13. Kang DH, Choi CW, Kim HW, et al. Predictors of upstage diagnosis after endoscopic resection of gastric low-grade dysplasia. Surg Endosc 2018;32:2732-2738.

14. Won CS, Cho MY, Kim HS, et al. Upgrade of lesions initially diagnosed as low-grade gastric dysplasia upon forceps biopsy following endoscopic resection. Gut Liver 2011;5:187193.

15. Maekawa A, Kato M, Nakamura T, et al. Incidence of gastric adenocarcinoma among lesions diagnosed as low-grade adenoma/dysplasia on endoscopic biopsy: a multicenter, prospective, observational study. Dig Endosc 2018;30:228-235.

16. de Vries AC, van Grieken NC, Looman CW, et al. Gastric cancer risk in patients with premalignant gastric lesions: a nationwide cohort study in the Netherlands. Gastroenterology 2008;134:945-952.

17. Kato M, Nishida T, Tsutsui S, et al. Endoscopic submucosal dissection as a treatment for gastric noninvasive neoplasia: a multicenter study by Osaka University ESD Study Group. J Gastroenterol 2011;46:325-331.

18. Park JS, Hong SJ, Han JP, et al. Early-stage gastric cancers represented as dysplasia in a previous forceps biopsy: the importance of clinical management. Dig Liver Dis 2013;45:170175.

19. Japanese Gastric Cancer Association. Japanese classification of gastric carcinoma: 3rd English edition. Gastric Cancer 2011;14:101-112.

20. The Paris endoscopic classification of superficial neoplastic lesions: esophagus, stomach, and colon: November 30 to December 1, 2002. Gastrointest Endosc 2003;58:S3-S43.

21. Kim YJ, Park JC, Kim JH, et al. Histologic diagnosis based on forceps biopsy is not adequate for determining endoscopic treatment of gastric adenomatous lesions. Endoscopy 2010;42:620-626.

22. Schlemper RJ, Itabashi M, Kato Y, et al. Differences in diagnostic criteria for gastric carcinoma between Japanese and western pathologists. Lancet 1997;349:1725-1729.

23. Cheung DY, Park SH. How to interpret the pathological report before and after endoscopic submucosal dissection of early gastric cancer. Clin Endosc 2016;49:327-331. 